\section{INTERVIEW MIT STEPHAN KIRSTE \\ OHNE DIE LITERATUR WÜRDE AM RECHT ETWAS WESENTLICHES FEHLEN}

\section{BegEgNung:Dieter AXT ${ }^{1}$}

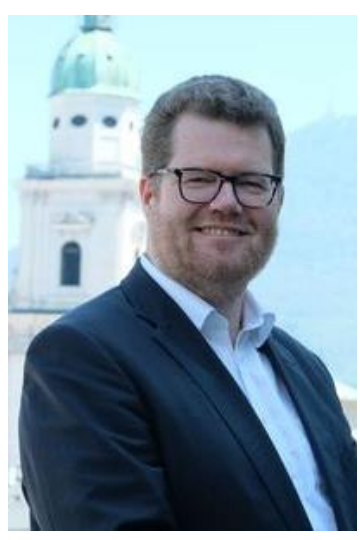

Die Abteilung SEÇÃo ENTREvistas beabsichtigt, einen möglichen Raum für Gespräch und Austausch zwischen wissenschaftlern der Rechtswissenschaften zu schaffen, die sich mit dem Verhältnis zwischen Recht und Literatur befassen.

In der vorliegenden Ausgahe haben wir die Freude, wieder eine Begegnung, diesmal mit Stephan Kirste, vorzustellen.

Stephan Kirste, geboren am 12. November 1962 in Oldenburg, ist ein deutscher Rechtswissenschaftler. Kirste hat an der Universität Salzburgeinen Lehrstuhl für Rechts-und Sozialphilosophie inne und ist Präsident der Deutschen Sektion der Internationalen Vereinigung für Rechts-und Sozialphilosophie.

1 Master-Abschluss in Öffentlichem Recht an der Universidade do Vale dos Sinos (UNISINOS). Junggeselle in Rechtswissenschaften an der Universidade Federal do Rio Grande do Sul (UFRGS). Drehbuchautor der Fernsehshow Direito \& Literatura (TV Justice - Brazil). Mitglied des brasilianischen Law and Literature Network (RDL). Redaktionsassistent der Anamorphosis - Internationales Journal für Recht und Literatur. Autor und Herausgeber des Verlages Le Chien. Porto Alegre, Brazil. CV Lattes: http://lattes.cnpq.br/1582390811392545. ORCID: https://orcid.org/o000-0002-09767326. E-mail: dieter@rdl.org.br 
Dieter Axt - In zahlreichen Universitäten in Brasilien ist die Behandlung des Themas Recht und Literatur noch am Anfang, wenn es sie überhaupt gibt. Welche Rolle spielt dieses Thema in Österreich? Was wird in Österreich zur Förderung der Beschäftigung mit diesem Thema gemacht, auch auf der Ebene der Institutionen (nicht nur im Bereich von einzelnen Initiativen).

An der Universität Graz wurde eine Tagung veranstaltet und dazu ein Sammelband herausgegeben, der von mir in der Zeitschrift für Öffentliches Recht rezensiert wurde: Christian Hiebaum; Susanne Knaller; Doris Pichler (Hg/eds), Recht und Literatur im Zwischenraum / Law and Literature In-Between. Aktuelle interund transdisziplinäre Zugänge / Contemporary Inter-and Transdisciplinary Approaches, Bielefeld 306 S. In: ZÖR 70 (2015), S. 643-645.

Wir werden im Oktober in Salzburg einen Workshop zu Legal Fictions veranstalten: https://www.uni-salzburg.at/index.php?id= 206876

Dieter Axt - Wird diesbezüglich in Österreich etwas anderes gemacht, was in Deutschland nicht praktiziert wird?

Nein, das würde ich nicht sagen. Vielleicht werden die Akzente etwas anders gesetzt.

Dieter Axt - Im Werk Poesie im Recht von 1815 schreibt Jacob Grimm: "Daß recht und poesie aus einem Bette aufgestanden waren, hält nicht schwer zu glauben". Sind Sie damit einvestanden, dass es kein Recht ohne Literatur geben kann? Weist diese berühmte Aussage von Grimm auf das Phänomen des Historismus eines Karl von Savigny hin?

Darüber könnte man einen Aufsatz schreiben. Grimm ist ja bei von Savigny in die Vorlesung in Marburg gegangen und blieb mit ihm befreundet, auch über die Brentanos. Ich würde nicht sagen, dass es kein Recht ohne Literatur geben kann. Aber ich würde ganz klar sagen, dass dem Recht Wesentliches fehlte, wenn es keine Literatur geben würde. Beide sind Formen von Kultur, beeinflussen sich gegenseitig und sind zu ihrem wechselseitigen Verständnis oft hilfreich. Das meint Grimm - und dem würde ich zustimmen. 
Dieter Axt - Sie waren schon öfters in Brasilien; Sie nahmen an Tagungen und an Buchvorstellungen teil. Letztes Jahr behandelten Sie auf der Tagung Recht und Literatur Gemeinsamkeiten und Unterschiede zwischen Recht und Literatur. Dabei erwähnten Sie Peter Goodrich, nach dem die Ausübung des Rechts die ständige Verneinung der literarischen Fähigkeiten verlangt. Vor kurzem behauptete Jeanne Gaakeer in der Zeitschrift ANAMORPHOSIS, dass "das Recht definitiv Erzählung" sei. Wie sehen Sie dieses dreifache Verhältnis zwischen Recht, Literatur und Narrativ (Erzählung) und welche sind die Grenzen zwichen dem Bereich der Gerichtsbarkeit und dem der Bühne und der Dichtung?

Eine Teilantwort habe ich gerade schon gegeben. Recht und Literatur sind Formen von Kultur. Allerdings unterschiedliche Formen. Am Wichtigsten: Recht ist verbindlich, Literatur nicht. Recht könnte sogar Literatur kanonisieren oder umgekehrt verbieten. Das kann Literatur nur durch Qualität, Begeisterungsfähigkeit usw. Schiller hat in wunderbarer Weise den Zusammenhang zwischen Literatur und Recht beschrieben, indem er das Recht auf die Bühne der Literatur und die Literatur vor die Schranken des Gerichts gezogen hat. Nussbaum hat in ihrem Buch über poetische Gerechtigkeit sehr schön beschrieben, wie Literatur ein Bewusstsein für eine tiefere, komplexere Gerechtigkeit schaffen kann, von der das Recht nur zu oft abstrahiert und abstrahieren muss.

Dieter Axt - Sie werden am 11. Oktober 2018 in Salzburg zusammen mit Prof. [Kristin] Albrecht die Veranstaltung Fiction and Law durchführen. Die Professorinen Greta Olson und Karen Petroski nehmen auch daran teil. Das Recht schafft in der Tat Fiktionen. Ich möchte Sie aber fragen, ob die Realität ohne eine gewisse Dosis Literatur überleben kann. Ist es ironisch zu denken, dass man durch die literarischen Fiktionen die rechtlichen Fiktionen besser verstehen könnte?

Hier nehme ich eine konstruktivistische Position ein: Wir konstruieren unsere soziale Realität. An diesem Prozess der Konstruktion der Realität nehmen Literatur und Recht teil, wenn auch in unterschiedlicher Weise. Beide sind in gewisser Hinsicht fiktiv, indem sie Gegenwelten schaffen zu den bisher vorgestellten Welten. Das Recht baut an einer besseren Welt, an einem neuen Jerusalem, durch seine Normen. Hierzu konstruiert es Positionen und Verfahren, die es ohne das Recht nicht gäbe. Das mag man fiktiv nennen. Teilweise schafft es sich auch eine eigene Natur. Das habe ich in meiner Dissertation über Recht und Zeit 
nachgewiesen. Die Literatur kann das viel phantasievoller, dramatischer, humorvoller, kurz: facettenreicher.

Dieter Axt - Gesellschaftsvertrag, volonté géneral, Gesetzlichkeit, Unparteilichkeit, Absicht des Gesetzgebers usw. Warum hat die vernünftige und säkulare Neuzeit so viele rechtliche Fiktionen geschaffen? In unserer Zeit sind auch neue Fiktionen entstanden?

Weil sie die Welt auf den Kopf, d.h. auf die Vernunft und nicht auf die Erfahrung, die Geschichte, soziale Zwänge stellt, wie Hegel gezeigt hat. Das Recht ist zutiefst idealistisch und deshalb arbeitet es auch mit solchen Idealisierungen. Das gilt auch für die Rechtstheorie, die hier Vorarbeit leistet.

Dieter Axt - Sie behaupten ein Meschenrecht auf die Demokratie, das in der Lage wäre, zur Konstruktion einer autonomen und emanzipierten bürgerlichen Gesellschaft beizutragen. Es geht dabei in der Tat um das Recht an der Begründung der universellen Rechte und Pflichten teilzunehmen. Könnten Sie Ihre These etwas ausführlicher erläutern? Welche sind die Grenzen dieser Teilnahme und welcher ist der Umfang der Universalisierung solches Rechts?

Soll der Mensch nicht ein Objekt von Herrschaft, sondern ein handelndes Subjekt sein, darf er nicht fremdbestimmten Normen unterworfen sein, sondern muss an ihrer Entstehung, Interpretation und Durchsetzung partizipieren können. Dass er als Subjekt bei aller Herrschaftsausübung geachtet werden soll, ist eine Forderung der Menschenwürde. Es gibt viele Formen der Partizipation. Es kann asymmetrische Partizipation nach Maßgabe der unterschiedlichen Betroffenheit oder gleichmäßige als politischer Bürger an den für alle in gleicher Weise geltenden Gesetzen geben. Wenn Partizipation ein Recht aus der Würde des Menschen ist, dann ist die gleiche Partizipation an den Gesetzen eine Unterform dieses Rechts. Dieses Recht kann das Menschenrecht auf Demokratie genannt werden. Wo immer also allgemeine Normen aufgestellt werden, hat der Mensch ein Recht auf Demokratie. Das ist auch im Völkerrecht der Fall. Also hat der Mensch auch hier ein Recht auf Demokratie. 
Dieter Axt - Sie beschäftigen sich intensiv mit dem Thema Menschenwürde. Es geht dabei um einen grundlegenden Begriff in der Geschichte der Zivilisation, der aus verschiedenen Gesichtspunkten behandelt werden kann: antropologischen, philosophischen und rechtlichen. Auch für die Literatur ist die Menschenwürde wichtig. Seit Antigone befassen sich zahlreiche Werke mit diesem Thema. Ist es möglich, einen Maßstab zur Menschenwürde zu etablieren?

"Maßstab zur Menschenwürde" - darf ich das so verstehen: Worin besteht der Maßstab, den die Menschenwürde aufstellt? - Ich habe das gerade schon gesagt: Der Mensch ist vielleicht das einzige Wesen, das sich zu dem machen kann, der es sein will (Pico della Mirandola). Durch diese Fähigkeit ist der Mensch Subjekt (Kant). Dass diese Fähigkeit aller Menschen - auch derjenigen, die sie aktuell nicht, nicht mehr oder noch nicht ausüben können - geschützt wird, fordert die Menschenwürde. Im Recht bedeutet es, dass der Mensch als Rechtssubjekt anerkannt werde, d.h. als Träger von Rechten und Pflichten. Der Mensch darf in einer Rechtsgemeinschaft kein Objekt von bloßer Gewalt sein. Vielmehr muss Herrschaft in den Bahnen des Rechts und das bedeutet unter grundsätzlicher Anerkennung der Rechtssubjektivität des Einzelnen ausgeübt werden. Ich meine, dass man in dieser Art einen säkularen Weise die Menschenwürde als rechtlichen Maßstab verstehen kann, ohne dass man auf starke philosophische oder religiöse Annahmen zurückgreifen muss.

Dieter Axt - Als Roland Barthes "den Tod des Autors" in einem bei der Aspen Mazagine veröffentlichten Artikel verkündet, beginnt eine neue Etappe der Interpretation, nämlich mit der Unterscheidung zwischen dem Willen des Autors und dem Willen des Gesetzes (des Textes). Inwiefern beeinflusst diese Einstellung von Barthes das Phänomen Interpretation? Ist der Autor tatsächlich tot?

Der Autor des Rechts ist nicht tot. Es ist der demokratische Gesetzgeber, die Parteien, die einen Vertrag schließen und der Richter, der ein Urteil spricht. Das Recht selbst hält sie im Demokratieprinzip, in der Privatautonomie und in der Rechtskraft von Urteilen lebendig. Es ist ihr jeweiliger Wille zu achten. Das hält sie - auch für die Interpretation am Leben. 
Dieter Axt - Zum Schluss, möchte ich Sie nach Ihren lieblingsten literarischen Werken befragen? Warum lesen wir immer wieder die Klassiker?

Das ist die schwerste Frage, weil ich viel zu wenig gute Literatur lese und viel zu viele Fachbücher. Ganz sicher aber gehören Goethes Faust und sein Wilhelm Meister dazu, Max Frisch Homo Faber und dann sehr viele philosophische Texte, besonders Hegels Grundlinien der Philosophie des Rechts, Platons Politeia und sein Siebter Brief dazu. Über weitere Texte müsste ich nachdenken. Ich lese diese Werke immer wieder als Inspirationsquellen für meine Arbeit und für mein Leben.

Dieter Axt - Möchten Sie über diese Fragen hinaus noch etwas sagen?

Ich danke Ihnen für diese Fragen, die mich ehren und zum Nachdenken angeregt haben! 